\title{
Combining Molecular Docking and Molecular Dynamics to Predict the Binding Modes of Flavonoid Derivatives with the Neuraminidase of the 2009 H1N1 Influenza A Virus
}

\author{
Shih-Jen Lu ${ }^{1,2, *}$ and Fok-Ching Chong ${ }^{1}$
}

1 Department of Electrical Engineering, National Taiwan University, Taipei 10617, Taiwan;

E-Mail: fcchong931@ hotmail.com

2 Department of Research and Development, BroadMaster Biotech Co., Ltd.: 7F., No.168-2, Liancheng Rd., Zhonghe Dist., New Taipei City 23553, Taiwan

* Author to whom correspondence should be addressed; E-Mail: d93921027@ntu.edu.tw;

Tel.: +886-266375859; Fax: +886-222428332.

Received: 9 January 2012; in revised form: 22 March 2012 / Accepted: 29 March 2012 /

Published: 10 April 2012

\begin{abstract}
Control of flavonoid derivatives inhibitors release through the inhibition of neuraminidase has been identified as a potential target for the treatment of H1N1 influenza disease. We have employed molecular dynamics simulation techniques to optimize the 2009 H1N1 influenza neuraminidase X-ray crystal structure. Molecular docking of the compounds revealed the possible binding mode. Our molecular dynamics simulations combined with the solvated interaction energies technique was applied to predict the docking models of the inhibitors in the binding pocket of the H1N1 influenza neuraminidase. In the simulations, the correlation of the predicted and experimental binding free energies of all 20 flavonoid derivatives inhibitors is satisfactory, as indicated by $R^{2}=0.75$.
\end{abstract}

Keywords: molecular dynamics; H1N1; neuraminidase; molecular docking

\section{Introduction}

Since March 2009, a new strain of the influenza A virus (H1N1) has rapidly spread to many countries from the initial outbreak in South America. In July 2009, the WHO (World Health Organization) declared that the spread of H1N1 influenza virus had become a serious global pandemic. Recently, the 
H1N1 influenza associated reports state that several mutation strains of H1N1 influenza A viruses are resistant to oseltamivir and zanamivir [1-3]. Influenza viruses have been classified by the antigenic properties of two glycoproteins, sixteen hemagglutinin proteins and nine neuraminidase proteins [4,5]. The hemagglutinin proteins play a role as antigens binding to the sialic acid receptor on the host cell surface, which aids the entry of the virus [6]. The function of the neuraminidase antigen is to cleave the terminal linkage of the sialic acid receptor, which results in the release of the progeny virions from the infected host cells. In addition, neuraminidase may have a function as importer facilitating the early process of the infection of lung epithelial cells by the influenza virus [7]. Because of its essential role in influenza virus replication and its highly conserved active sites, neuraminidase has been an attractive target for the development of novel anti-influenza drugs [8-14].

In recent years, several flavonoids have been reported as showing anti-influenza virus activity by inhibiting neuraminidase [15-19]. Flavonoids are low molecular weight compounds that are widespread in the plant kingdom. Those compounds have been shown to possess several biological effects in mammals [20-22]. In particular in vitro studies, scientists report that flavonoids may inhibit several enzymes related to the cardiovascular system [23-27], especially inhibiting the Matrix Metalloproteinases (MMPs) [28]. Therefore, using flavonoids as antivirals should be carefully considered in addition to these other proposed activities. In general, flavonoids are interesting molecules combining an aromatic nature with several hydrophilic groups. These aromatic interactions play a key role in protein-protein and protein-ligand interactions [29-31]. The hydrophilic nature (hydroxyl $(\mathrm{OH})$ functional group of flavonoids/water molecules) of the falvonoids shows that water displacement is key for determining ligand affinity [32-36].

Scientists also report that the flavonoid derivatives can efficiently inhibit the activity of H1N1 neuraminidase [37]. To reveal the inhibition mechanism of flavonoid derivatives on H1N1 neuraminidase, a knowledge of the three-dimensional structure of H1N1 neuraminidase is indispensable. Since H1N1 neuraminidase structures have been determined by X-ray experiments [5,38], we chose the structure (PBD ID: 3NSS) as the target structure for these studies.

In this study, the 20 flavonoid derivatives (2,3-dihydrobenzofuran and 5,7-dihydroxychromen-4-one backbones) and their experimental biological binding affinities [37,39] were chosen to simulate H1N1 neuraminidase pharmacological activities; these inhibitors are listed in Table S1. The transfer function [40] $\left(\Delta G\right.$ bind $=-R T \ln \left(\mathrm{IC}_{50}\right)$ ) is used to transfer the experimental values $\left(\mathrm{IC}_{50}\right)$ to the experimental binding free energies values; these experimental values are listed in Table S1. Molecular docking, molecular dynamics simulations (MD), and binding free energies calculations were used to gain further insight into the binding interactions between the $2009 \mathrm{H} 1 \mathrm{~N} 1$ neuraminidase and the 20 flavonoid derivatives inhibitors.

\section{Results and Discussion}

\subsection{Molecular Docking and MD Simulation}

The 20 flavonoid derivatives were docked into the H1N1 neuraminidase structure. Over the 10-ns MD trajectories of the H1N1 neuraminidase with tip3 water molecules and flavonoid derivatives, the overall structure of both complexes appeared to be equilibrated after 324 ps. Here, we show the 
RMSD profiles of 20 flavonoid derivatives (Figure 1) and the snapshot (Figure 2) of the complex system of the flavonoid derivatives 1 . The RMSD values of 20 flavonoids stay within $4 \AA$.

Figure 1. RMSD profiles of 20 flavonoid derivatives.

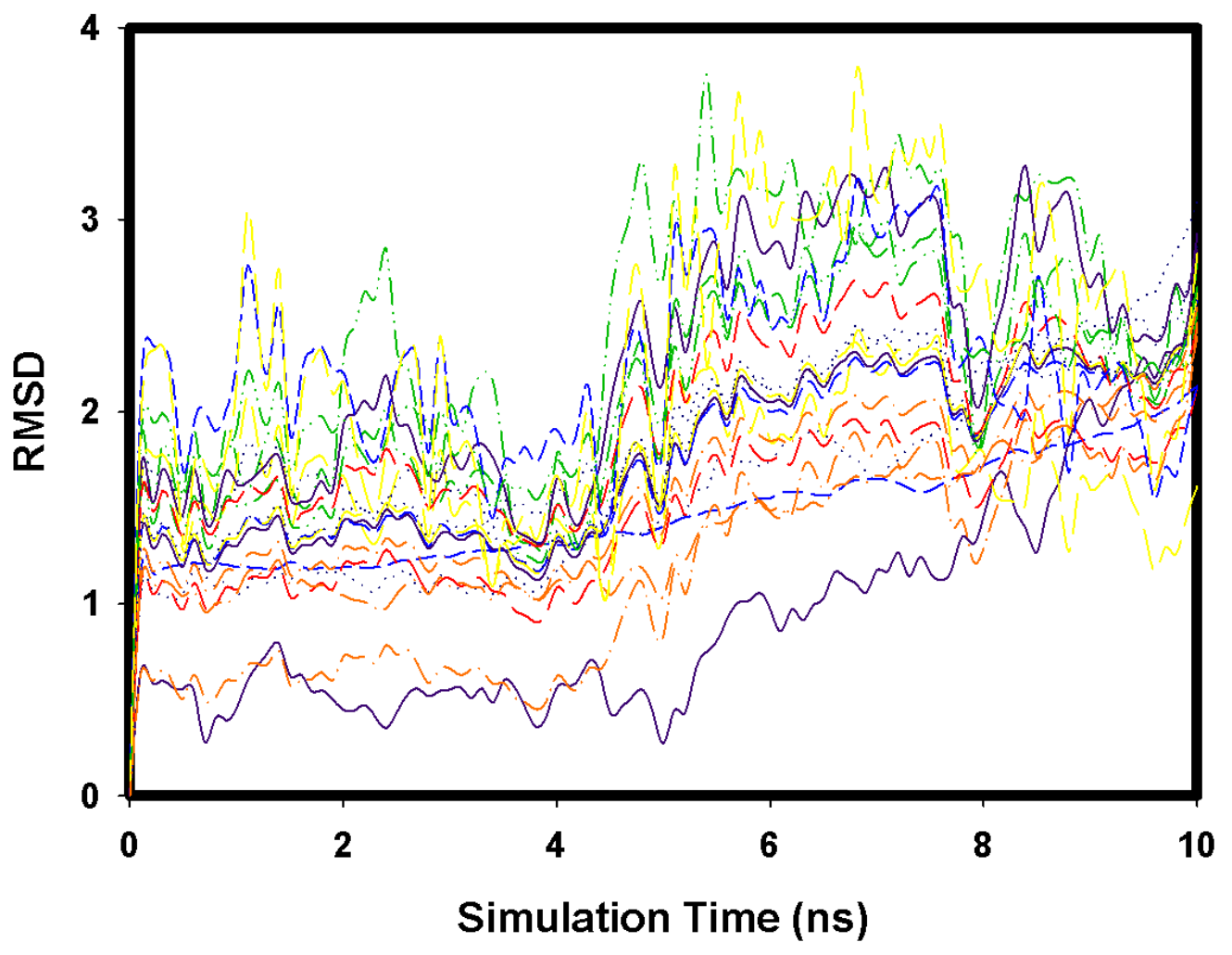

inhibitor 1 inhibitor 2

inhibitor 3

inhibitor 4

inhibitor 5

inhibitor 6

inhibitor 7

inhibitor 8

inhibitor 9

inhibitor 10

inhibitor 11

inhibitor 12

inhibitor 13

inhibitor 14

inhibitor 15

inhibitor 16

inhibitor 17

inhibitor 18

inhibitor 19

inhibitor 20

Figure 2. The snapshot of the 2009 H1N1 neuraminidase of the inhibitor 1 .

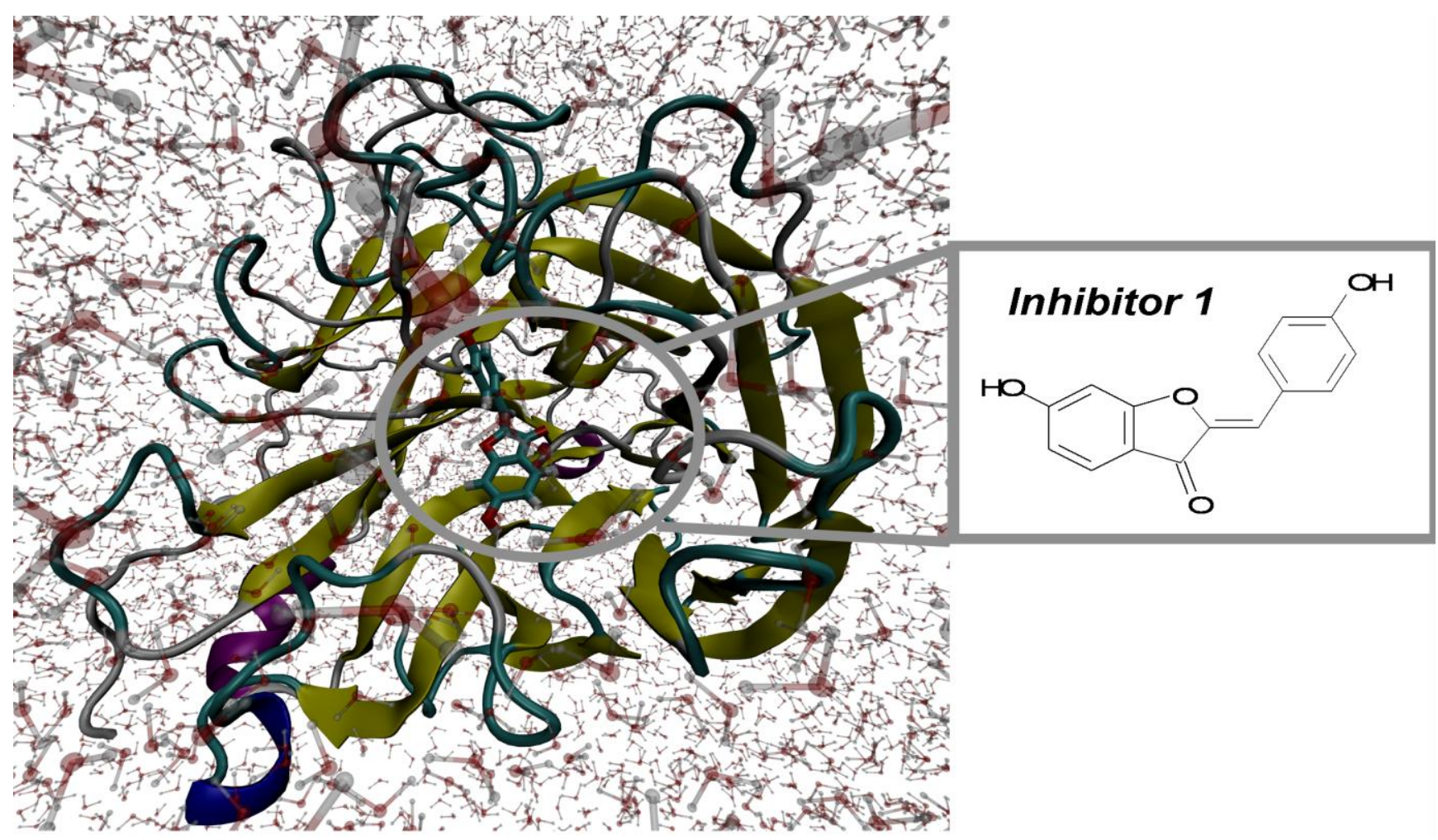




\subsection{Key Residues of 2009 H1N1 Neuraminidase}

The study of these 20 compounds has revealed that the amino residues can frequently interact with flavonoid inhibitors in the H1N1 neuraminidase binding site, and that these residues are responsible for the selectivity of flavonoid inhibitors. The results of our simulations are listed in Table 1 and Figure S1-S20. The inhibitors 1-3 and 14 (Table 1) belong to the 2,3-dihydrobenzofuran backbone inhibitors and the others belong to the 5,7-dihydroxychromen-4-one backbone inhibitors. In the 2,3-dihydrobenzofuran backbone inhibitors (inhibitor 1-3 and 14), Asn295,Glu119, Glu277, Thr226, Trp179 can form hydrogen bonds in the 2009 H1N1 neuraminidase/flavonoids complex structures and Asn295 most frequently forms the hydrogen bonds. Only Tyr402 has non-bonding interactions with inhibitor 1 (Figure S1). In the 5,7-dihydroxychromen-4-one backbone inhibitors (inhibitor 4-13 and 15-20), Arg152, Asn295, Asn325, Asn344, Asp151, Asp294, Glu119, Glu228, Glu277, Ser180, Ser247, Ser366, Ser367, Thr226, Trp179, Tyr402 and Val346 can form hydrogen bonds in the complex structures and Glu228 most frequently forms the hydrogen bonds. Arg368, Ile223, Pro326 and Trp179 have non-bonding interactions with the backbone inhibitors (Figure S7, 16 and 19). The overall results of our simulations suggest that Arg152, Asn295, Asn325, Asn344, Asp151, Asp295, Glu119, Glu228, Glu277, Ser180, Ser247, Ser366, Ser367, Thr226, Trp179, Tyr402 and Val346 can form hydrogen bonds between the $2009 \mathrm{H} 1 \mathrm{~N} 1$ neuraminidase and flavonoid derivatives. Moreover, our simulations indicate that Arg368, Ile223, Pro326 and Trp179 have non-bonding interactions with these derivatives. The non-bonding interactions of the 2009 H1N1 neuraminidase/flavonoid complex structures only occurred in inhibitor 1, 7, 16 and 19 simulations. While six residues (Arg152, Asn295, Glu228, Glu277 Trp179 and Val346) more often formed the hydrogen bonds of the complex structures, Asn295 most frequently formed the hydrogen bonds.

Table 1. Important results: Important residues of the 2009 H1N1 neuraminidase from the molecular docking and molecular dynamics (MD) simulations.

\begin{tabular}{clc}
\hline Inhibitors & Hydrogen bonding-related residues & Non-bonding contact-related residues \\
\hline 1(A) & Trp179, Thr226, Asn295 & Tyr402 \\
2(A) & Glu277, Asn295 & Null \\
3(A) & Glu119, Asn295 & Null \\
4(B) & Glu119, Ser180, Glu228, Asp294, Val346 & Null \\
5(B) & Val346 & Null \\
6(B) & Arg152, Glu228, Glu277 Asn295 & Null \\
7(B) & Asn295 & Trp179, Ile223 \\
8(B) & Glu119, Ser180, Glu228, Asp294, Val346 & Null \\
9(B) & Arg152, Glu228, Val346 & Null \\
10(B) & Ser180, Thr226, Glu228, Asp294 & Null \\
11(B) & Glu228, Glu277 & Null \\
12(B) & Asp151, Glu277, Asn325, Val346, Ser366 & Null \\
13(B) & Trp179, Asn295 & Null \\
14(A) & Asn295 & Null \\
15(B) & Arg152, Trp179, Asn295 & Null \\
16(B) & Glu119, Asp151, Ser366, Ser367, Tyr402 & Ile223, Arg368 \\
\hline
\end{tabular}


Table 1. Cont.

\begin{tabular}{clc}
\hline Inhibitors & Hydrogen bonding-related residues & Non-bonding contact-related residues \\
\hline 17(B) & Trp179 & Null \\
18(B) & Asp151, Arg152, Asn344 & Null \\
19(B) & Asp151, Trp179, Glu228, Asn295 & Pro326 \\
20(B) & Arg152, Trp179, Ser247, Glu277 & Null \\
\hline
\end{tabular}

A: 2,3-dihydrobenzofuran backbone and B: 5,7-dihydroxychromen-4-one backbone.

\subsection{Flavonoid Derivatives Binding Free Energies}

The 2009 H1N1 neuraminidase/flavonoid (inhibitors 1-20) complex structures and the Tip3 water solvent box were constructed using the Autodock Vina docking and AmberTools 1.5 programs. The binding free energies of each inhibitor were obtained from the 10-ns MD simulation and the SIE method, with both processes using the same parameters. All the results are listed in Table 2. In the simulations, the predicted binding free energies of the 20 inhibitors was in good agreement with the experimental results (Figure 3), with the correlation coefficient being 0.75 .

Table 2. Binding free energies for the 2009 H1N1 neuraminidase/flavonoid (inhibitors 1-20) complex structures by the SIE method.

\begin{tabular}{ccccccc}
\hline Inhibitors & \multicolumn{7}{c}{ Energy (kcal/mol) } \\
\cline { 2 - 6 } & $E_{C}$ & $E_{\text {wwd }}$ & $\Delta M S A$ & $\Delta G_{\text {bind }}^{R}$ & $\Delta G_{\text {bind }}($ SIE) & $\Delta G_{\text {bind }}$ (Experiment) \\
\hline $\mathbf{1}$ & -20.7 & -9.95 & 6.12 & -6.98 & -6.825 & -7.002 \\
$\mathbf{2}$ & -20.9 & -7.36 & 5.29 & -5.64 & -6.435 & -6.714 \\
$\mathbf{3}$ & -15.2 & -6.69 & 4.83 & -5.71 & -5.771 & -6.518 \\
$\mathbf{4}$ & -20.3 & -5.31 & 5.31 & -5.91 & -6.186 & -6.430 \\
$\mathbf{5}$ & -16.9 & -6.12 & 5.81 & -7.41 & -6.071 & -6.296 \\
$\mathbf{6}$ & -24.1 & -7.26 & 6.43 & -6.39 & -6.837 & -6.265 \\
$\mathbf{7}$ & -20.9 & -7.36 & 5.11 & -5.43 & -6.413 & -6.014 \\
$\mathbf{8}$ & -16.9 & -4.81 & 6.57 & -5.94 & -5.779 & -5.847 \\
$\mathbf{9}$ & -12.9 & -4.64 & 5.11 & -5.33 & -5.277 & -5.773 \\
$\mathbf{1 0}$ & -15.4 & -4.73 & 5.59 & -6.11 & -5.633 & -5.717 \\
$\mathbf{1 1}$ & -13.9 & -4.91 & 6.19 & -6.82 & -5.567 & -5.629 \\
$\mathbf{1 2}$ & -12.2 & -6.71 & 3.91 & -6.08 & -5.503 & -5.608 \\
$\mathbf{1 3}$ & -11.4 & -5.73 & 4.41 & -4.91 & -5.193 & -5.519 \\
$\mathbf{1 4}$ & -12.4 & -5.12 & 5.25 & -5.51 & -5.291 & -5.489 \\
$\mathbf{1 5}$ & -15.4 & -4.73 & 5.65 & -6.41 & -5.766 & -5.450 \\
$\mathbf{1 6}$ & -9.3 & -8.73 & 6.81 & -5.56 & -5.353 & -5.343 \\
$\mathbf{1 7}$ & -12.9 & -4.32 & 4.81 & -5.09 & -5.221 & -5.226 \\
$\mathbf{1 8}$ & -12.7 & -4.21 & 4.61 & -4.84 & -5.163 & -5.225 \\
$\mathbf{1 9}$ & -11.6 & -4.29 & 4.82 & -5.11 & -5.084 & -5.199 \\
$\mathbf{2 0}$ & -10.1 & -4.33 & 4.99 & -4.97 & -4.916 & -5.007 \\
\hline & & & & & & \\
\hline
\end{tabular}


Figure 3. Predicted binding free energies versus experimentally determined binding free energies of the 20 inhibitors. The correlation constant $\left(R^{2}\right)$ is equal to 0.75 . The inhibitors 1-3 and 14 (red) belong to the 2,3-dihydrobenzofuran backbone inhibitors and the others belong to the 5,7-dihydroxychromen-4-one backbone inhibitors (black).

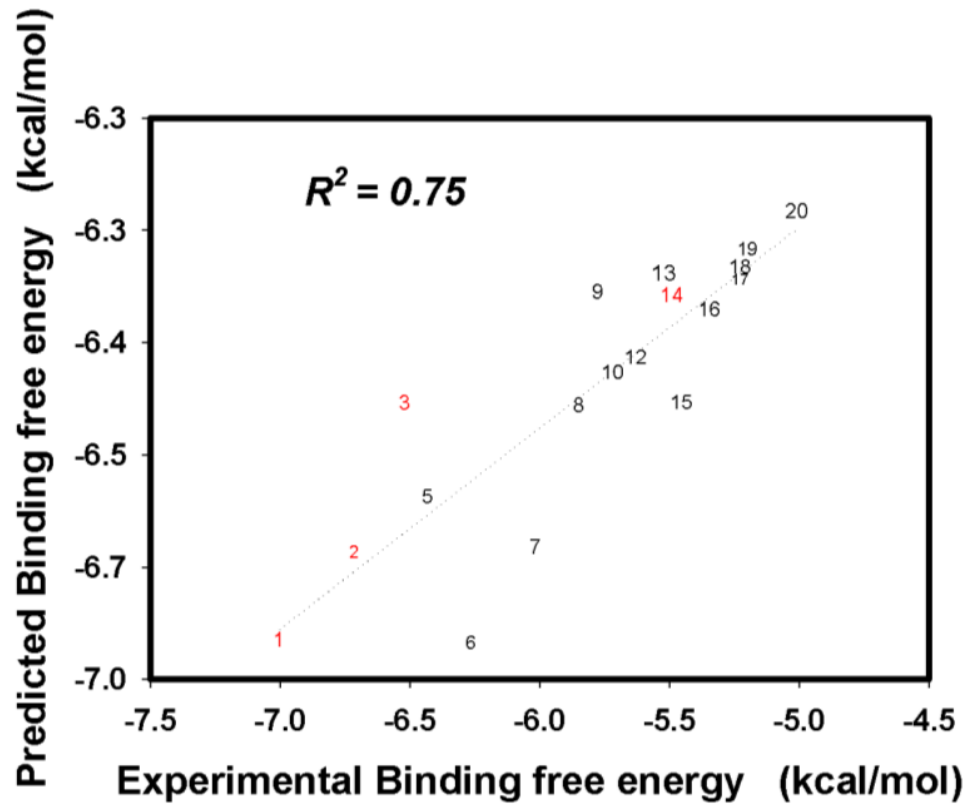

\subsection{Free Energies Contribution Analysis of Hydrophobic and Hydrophilic Nature of 20 Flavonoids}

The hydrophobic and hydrophilic nature of 20 flavonoids was individually traced down the binding affinities with the important residue regions analyzed by the ligplot program (Arg152, Asn295, Asn325, Asn344, Asp151, Asp295, Glu119, Glu228, Glu277, Ser180, Ser247, Ser366, Ser367, Thr226, Trp179, Tyr402), and the water molecules (within a $10 \AA$ radius of 20 flavonoids). All the results are listed in Tables S2,3 and Figures S21,22. For the hydrophobic nature analysis (aromatic groups), the two residues (Trp179 and Tyr402) have obvious binding affinities to the inhibitors. The contributions to Trp179 and Tyr402 binding free energies are $-1.121 \sim-2.961$ and $-1.665 \sim-3.143 \mathrm{kcal} / \mathrm{mol}$, respectively. For the hydrophilic nature analysis, the three elements (side chain of nitrogen atoms/Arg152, side chain of nitrogen and oxygen atoms/Asn295 and the water molecules within $10 \AA$ of inhibitors) have obvious binding affinities to the inhibitors. Those contributions to Arg152, Asn295 and water molecules binding free energies are $-0.507 \sim-2.733,-0.185 \sim-2.247$ and $-0.6 \sim-2.6 \mathrm{kcal} / \mathrm{mol}$, respectively. Figure S23 shows that the more hydroxyl function groups are present, the higher the binding affinities of the inhibitors.

\section{Materials and Methods}

\subsection{Molecular Docking}

All flavonoid derivatives inhibitors were constructed and minimized using VEGA ZZ [41] and ISIS/DRAW [42] programs. We aligned the H1N1 neuraminidase structure (PBD ID: 3NSS) and the template (PDB ID: 3B7E) [43], and then used the template structure drug (zanamivir) as a template to generate the active site of the H1N1 neuraminidase structure. Next, the Autodock vina docking 
program [44] was used to dock the 20 flavonoid derivatives inhibitors into the active site of the H1N1 neuraminidase structure, which was defined as all residues within $0.15 \mathrm{~nm}$ from alignment with the template structure drug. Autodock vina is a fast and accurate way to dock small compounds into fixed protein binding sites, utilizing NNscore [45] and several types of genetic algorithms. Four thousand conformations were obtained from docking for the 20 inhibitors, and these then scored by NNscore. The conformations of the best NNscores were then selected for subsequent MD simulations.

\subsection{Molecular Dynamics Simulation}

Calculations were performed with the NAMD molecular dynamics software [46] using the AMBER FF99 all-hydrogen amino acid and general amber force field (GAFF) parameters. The GAFF partial atomic charges are often based on the RESP fitting procedure of the electrostatic potential obtained at the HF/6-31G(d,p) level of theory [47]. Those levels of theory overestimate the gas-phase partial atomic charges giving rise to an effectively polarized force field. The geometries of the 20 flavonoid derivatives inhibitors were fully optimized and their electrostatic potentials were obtained using a single-point calculation; both operations were carried out at the HF level with the 6-31 G(d,p) basis set using the US GAMESS [48] program. Subsequently, their partial charges were obtained by the restrained electrostatic potential (RESP) using R.E.D tools [49]. From the docking simulations, the complex structures were inserted into the tip3p water box. All MD simulations were performed in the NVT ensemble (temperature equal to $310 \mathrm{~K}$ ), unless noted, using a Verlet integrator, an integration time step of $0.002 \mathrm{ps}$, and SHAKE [50] data for all covalent bonds involving hydrogen atoms. In electrostatic interactions, atom-based truncation was undertaken using the PME method. In addition, the switch van der Waals functions were also used with a $1.8 \mathrm{~nm}$ cutoff for atom-pair lists. The complex structures were minimized for 20,000 conjugate gradient steps. The minimized complex structures were then subjected to a $10 \mathrm{~ns}$ isothermal, constant volume MD simulation. The MD simulation trajectories were converted to the Amber type coordinates by the AmberTools 1.5 program [51]. All MD simulation results were used to initiate the functionally important residues and the binding free energies calculations.

\subsection{Functionally Important Residues of H1N1 Influenza Neuraminidase}

In a diseased target proteins, not every residue is equally important. Some residues are essential for important enzymatic functions and protein structure stability. Thus identification of functionally important residues can provide a clear insight into the structural aspects of H1N1 2009 influenza neuraminidase. In this work, the structure-based approach was applied to identify functionally important residues, while MD simulations were used to identify the residues involved in the binding pocket. In the MD simulations, the important residues regions were analyzed by the ligplot program [52]. The results of functional important residues were applied to binding free energies calculations.

\subsection{Binding Free Energies Calculations (Solvated Interaction Energies Method)}

The binding free energies calculations were performed by the solvated interaction energies method (SIE). The binding free energies between receptor and inhibitors were calculated for snapshot 
structures taken from the MD trajectory of the system. From the 10 ns protein-ligand MD trajectories, 100 snapshots were taken at regular intervals for the binding energies analyses. The SIE function [53] to estimate the binding free energies is written as:

$$
\begin{aligned}
\Delta G_{\text {bind }}\left(\rho, D_{i n}, \alpha, \gamma, C\right)= & \alpha \times\left[E_{c}\left(D_{i n}\right)+\Delta G_{b i n d}^{R}\left(\rho, D_{i n}\right)\right. \\
& \left.+E_{v d w}+\gamma \Delta M S A(\rho)\right]+C
\end{aligned}
$$

where $E_{C}$ and $E_{v d w}$ are the intermolecular Coulomb and van der Waals interaction energies in the bound state, respectively. These values were calculated using the AMBER molecular mechanics force field (FF99) with an optimized dielectric constant. $\Delta G_{\text {bind }}^{R}$ is the change in the reaction field energies between the bound and free states and is calculated by solving the Poisson equation with the boundary element method program, BRI BEM, and using a molecular surface generated with a variable-radius solvent probe. The $\triangle \mathrm{MSA}$ term is the change in the molecular surface area upon binding. The following parameters are calibrated by fitting to the absolute binding free energies for a set of protein-ligand complexes: AMBER van der Waals radii linear scaling coefficient $(\rho)$, the solute interior dielectric constant (Din), the molecular surface area coefficient $(\gamma)$, the global proportionality coefficient related to the loss of configurational entropy upon binding $(\alpha)$, and a constant $(C)$. The optimized values of these parameters are $\alpha=0.1048$, Din $=2.25, \rho=1.1, \gamma=0.0129 \mathrm{kcal} /\left(\mathrm{mol} \AA^{2}\right)$, and $C=-2.89 \mathrm{kcal} / \mathrm{mol}$. The SIE calculations were carried out with the program sietraj [53]. The hydrophobic (non-hydroxyl group) and hydrophilic (hydroxyl group) nature of flavonoids can affect the binding abilities [29-31]. Thus these natures were individually traced down to the binding affinities with the whole H1N1 neuraminidase, the important residue regions analyzed by the ligplot program (hydrophilic and hydrophobic parts listed in Table S4), and the water molecules (within a $10 \AA$ radius of 20 flavonoids).

\section{Conclusions}

In this article, we used the Autodock vina program, tip3 water solvent model, MD simulations techniques, and the SIE method to predict the binding modes in which a series of flavonoid derivatives inhibitors interact with the 2009 H1N1 neuraminidase. From our simulations results, the correlation coefficient between the predicted binding free energies and experimental values of the 20 inhibitors is equal to 0.75 . In the 2,3-dihydrobenzofuran backbone derivatives inhibitors (inhibitor 1-3 and 14), Asn295 forms the hydrogen bonds most frequently. In the 5,7-dihydroxychromen-4-one backbone derivatives inhibitors (inhibitor 4-13 and 15-20), Glu228 forms the hydrogen bonds most frequently.

The overall results of our simulations indicate that Arg152, Asn295, Asn325, Asn344, Asp151, Asp294, Glu119, Glu228, Glu277, Ser180, Ser247, Ser366, Ser367, Thr226, Trp179, Tyr402 and Val346 can form hydrogen bonds between the 2009 H1N1 neuraminidase and flavonoid derivatives. While six residues (Arg152, Asn295, Glu228, Glu277 Trp179 and Val346) more often form the hydrogen bonds of the complex structures, Asn295 forms the hydrogen bonds most frequently. In the free energies contribution analysis, the two residues (the indole group of Trp179 and the benzene group of Tyr402) have obvious binding affinities to the hydrophobic nature (aromatic-aromatic interactions) of the 20 inhibitors ((2Z)-2-benzylidene-3H-benzofuran, 2-phenyl-4H-chromene and benzene aromatic groups), and the three elements (the guanidine group of Arg152, the acetamide 
group of Asn295 and the water molecules within $10 \AA$ of inhibitors) have obvious binding affinities to the hydrophilic nature of the 20 inhibitors ( $-\mathrm{OH}$ and $=\mathrm{O}$ groups). Figure $\mathrm{S} 23$ also shows that the more hydroxyl and oxygen function groups are present, the greater the number of binding affinities of the inhibitors. Therefore, our approach theoretically suggests that the four residues Arg152, Trp179, Asn295 and Tyr402 are responsible for the selectivity of the flavonoid derivatives.

\section{Supporting Information}

The $2 \mathrm{D}$ structures, experimental $\mathrm{IC}_{50}$ and experimental binding free energies of 20 flavonoid derivatives are listed in Table S1. The functional residues analysis of the 20 flavonoid derivatives are listed in Figures S1-S20. Binding free energies analysis of hydrophobic/hydrophilic nature of 20 flavonoids are listed in Tables S2-S3 and Figures S21-S23.

\section{Acknowledgments}

This work was supported by the National Taiwan University.

\section{References}

1. Ginting, T.E.; Shinya, K.; Kyan, Y.; Makino, A.; Matsumoto, N.; Kaneda, S.; Kawaoka, Y. Amino acid changes in hemagglutinin contribute to the replication of oseltamivir-resistant H1N1 influenza viruses. J. Virol. 2011, doi:10.1128/JVI.06085-11JVI.06085-11.

2. Van der Vries, E.; Veldhuis Kroeze, E.J.; Stittelaar, K.J.; Linster, M.; van der Linden, A.; Schrauwen, E.J.A.; Leijten, L.M.; van Amerongen, G.; Schutten, M.; Kuiken, T.; et al. Multidrug resistant $2009 \mathrm{~A} / \mathrm{H} 1 \mathrm{~N} 1$ influenza clinical isolate with a neuraminidase I223R mutation retains its virulence and transmissibility in ferrets. PLoS Pathog. 2011, 7, doi:10.1371/journal.ppat.1002276.

3. Abed, Y.; Boivin, G.; Yoshida, R.; Kodama, M.; Hernandez, J.E. Parenteral peramivir treatment for oseltamivir-resistant 2009 pandemic influenza A H1N1 viruses. J. Infect. Dis. 2011, 204, 1641-1642.

4. Nomura, N.; Sakoda, Y.; Endo, M.; Yoshida, H.; Yamamoto, N.; Okamatsu, M.; Sakurai, K.; Hoang, N.; Nguyen, L.; Chu, H.; et al. Characterization of avian influenza viruses isolated from domestic ducks in Vietnam in 2009 and 2010. Arch. Virol. 2012, 157, 247-257.

5. Vavricka, C.J.; Li, Q.; Wu, Y.; Qi, J.; Wang, M.; Liu, Y.; Gao, F.; Liu, J.; Feng, E.; He, J.; et al. Structural and functional analysis of laninamivir and its octanoate prodrug reveals group specific mechanisms for influenza NA inhibition. PLoS Pathog. 2011, 7, doi:10.1371/journal.ppat.1002249.

6. Takeda, M.; Leser, G.P.; Russell, C.J.; Lamb, R.A. Influenza virus hemagglutinin concentrates in lipid raft microdomains for efficient viral fusion. Proc. Natl. Acad. Sci. USA 2003, 100, 14610-14617.

7. Jennifer, L.M.-B. Resistance of influenza viruses to neuraminidase inhibitors-a review. Antivir. Res. 2000, 47, 1-17. 
8. Wang, S.-Q.; Du, Q.-S.; Chou, K.-C. Study of drug resistance of chicken influenza A virus (H5N1) from homology-modeled 3D structures of neuraminidases. Biochem. Biophys. Res. Commun. 2007, 354, 634-640.

9. Wang, Y.-T.; Chan, C.-H.; Su, Z.-Y.; Chen, C.-L. Homology modeling, docking, and molecular dynamics reveal HR1039 as a potent inhibitor of 2009 A(H1N1) influenza neuraminidase. Biophys. Chem. 2010, 147, 74-80.

10. Liu, H.; Yao, X.; Wang, C.; Han, J. In silico identification of the potential drug resistance sites over 2009 influenza A (H1N1) virus neuraminidase. Mol. Pharm. 2010, 7, 894-904.

11. Amaro, R.E.; Swift, R.V.; Votapka, L.; Li, W.W.; Walker, R.C.; Bush, R.M. Mechanism of 150cavity formation in influenza neuraminidase. Nat. Commun. 2011, 2, doi:10.1038/ncomms 1390.

12. Pan, D.; Sun, H.; Bai, C.; Shen, Y.; Jin, N.; Liu, H.; Yao, X. Prediction of zanamivir efficiency over the possible 2009 Influenza A (H1N1) mutants by multiple molecular dynamics simulations and free energy calculations. J. Mol. Model. 2011, 17, 2465-2473.

13. Chavan, S.; Bhayye, S.; Sobhia, M. Molecular dynamics directed CoMFA studies on carbocyclic neuraminidase inhibitors. Mol. Divers. 2011, 15, 979-987.

14. Mai, B.K.; Li, M.S. Neuraminidase inhibitor R-125489-A promising drug for treating influenza virus: Steered molecular dynamics approach. Biochem. Biophys. Res. Commun. 2011, 410, 688-691.

15. Liu, A.-L.; Wang, H.-D.; Lee, S.M.; Wang, Y.-T.; Du, G.-H. Structure-activity relationship of flavonoids as influenza virus neuraminidase inhibitors and their in vitro anti-viral activities. Bioorg. Med. Chem. 2008, 16, 7141-7147.

16. Wei, F.; Ma, S.-C.; Ma, L.-Y.; But, P.P.-H.; Lin, R.-C.; Khan, I.A. Antiviral flavonoids from the seeds of aesculus chinensis. J. Nat. Prod. 2004, 67, 650-653.

17. Li, Y.; Leung, K.-T.; Yao, F.; Ooi, L.S.M.; Ooi, V.E.C. Antiviral flavans from the leaves of pithecellobium clypearia. J. Nat. Prod. 2006, 69, 833-835.

18. Miki, K.; Nagai, T.; Nakamura, T.; Tuji, M.; Koyama, K.; Kinoshita, K.; Furuhata, K.; Yamada, H.; Takahashi, K. Synthesis and evaluation of influenza virus sialidase inhibitory activity of hinokiflavone-sialic acid conjugates. Heterocycles 2008, 75, 879-885.

19. Miki, K.; Nagai, T.; Suzuki, K.; Tsujimura, R.; Koyama, K.; Kinoshita, K.; Furuhata, K.; Yamada, H.; Takahashi, K. Anti-influenza virus activity of biflavonoids. Bioorg. Med. Chem. Lett. 2007, 17, 772-775.

20. Maron, D. Flavonoids for reduction of atherosclerotic risk. Curr. Atheroscler. Rep. 2004, 6, 73-78.

21. Huxley, R.R.; Neil, H.A.W. The relation between dietary flavonol intake and coronary heart disease mortality: A meta-analysis of prospective cohort studies. Eur. J. Clin. Nutr. 2003, 57, 904-908.

22. Hodgson, J.M.; Croft, K.D. Dietary flavonoids: Effects on endothelial function and blood pressure. J. Sci. Food Agric. 2006, 86, 2492-2498.

23. Ruiz-Ortega, M.; Esteban, V.; Egido, J. The regulation of the inflammatory response through nuclear factor- $\kappa \mathrm{B}$ pathway by angiotensin IV extends the role of the renin angiotensin system in cardiovascular diseases. Trends Cardiovasc. Med. 2007, 17, 19-25.

24. Pignatelli, P.; Pulcinelli, F.M.; Celestini, A.; Lenti, L.; Ghiselli, A.; Gazzaniga, P.P.; Violi, F. The flavonoids quercetin and catechin synergistically inhibit platelet function by antagonizing the intracellular production of hydrogen peroxide. Am. J. Clin. Nutr. 2000, 72, 1150-1155. 
25. Hirvonen, T.; Pietinen, P.; Virtanen, M.; Ovaskainen, M.-L.; Häkkinen, S.; Albanes, D.; Virtamo, J. Intake of flavonols and flavones and risk of coronary heart disease in male smokers. Epidemiology 2001, 12, 62-67.

26. Raso, G.M.; Meli, R.; Di Carlo, G.; Pacilio, M.; di Carlo, R. Inhibition of inducible nitric oxide synthase and cyclooxygenase-2 expression by flavonoids in macrophage J774A.1. Life Sci. 2001, 68, 921-931.

27. Welton, A.F.; Hurley, J.; Will, P. Flavonoids and arachidonic acid metabolism. Prog. Clin. Biol. Res. 1988, 280, 301-312.

28. Saragusti, A.C.; Ortega, M.G.; Cabrera, J.L.; Estrin, D.A.; Marti, M.A.; Chiabrando, G.A. Inhibitory effect of quercetin on matrix metalloproteinase 9 activity Molecular mechanism and structure-activity relationship of the flavonoid-enzyme interaction. Eur. J. Pharmacol. 2010, 644, 138-145.

29. Chin, D.; Means, A.R. Calmodulin: A prototypical calcium sensor. Trends Cell Biol. 2000, 10, 322-328.

30. Lanzarotti, E.; Biekofsky, R.R.; Estrin, D.A.; Marti, M.A.; Turjanski, A.G. Aromatic-Aromatic interactions in proteins: Beyond the dimer. J. Chem. Inf. Model. 2011, 51, 1623-1633.

31. Espinoza-Fonseca, L.M.; García-Machorro, J. Aromatic-aromatic interactions in the formation of the MDM2-p53 complex. Biochem. Biophys. Res. Commun. 2008, 370, 547-551.

32. Gauto, D.F.; Di Lella, S.; Guardia, C.M.A.; Estrin, D.A.; Martí, M.A. Carbohydrate-binding proteins: Dissecting ligand structures through solvent environment occupancy. J. Phys. Chem. B 2009, 113, 8717-8724.

33. Abel, R.; Young, T.; Farid, R.; Berne, B.J.; Friesner, R.A. Role of the active-site solvent in the thermodynamics of factor Xa ligand binding. J. Am. Chem. Soc. 2008, 130, 2817-2831.

34. Michel, J.; Tirado-Rives, J.; Jorgensen, W.L. Energetics of displacing water molecules from protein binding sites: Consequences for ligand optimization. J. Am. Chem. Soc. 2009, 131, 15403-15411.

35. Di Lella, S.; Martí, M.A.; Álvarez, R.M.S.; Estrin, D.A.; Ricci, J.C.D. Characterization of the galectin-1 carbohydrate recognition domain in terms of solvent occupancy. J. Phys. Chem. B 2007, $111,7360-7366$.

36. Gauto, D.F.; di Lella, S.; Estrin, D.A.; Monaco, H.L.; Martí, M.A. Structural basis for ligand recognition in a mushroom lectin: Solvent structure as specificity predictor. Carbohydr. Res. 2011, 346, 939-948.

37. Mercader, A.G.; Pomilio, A.B. QSAR study of flavonoids and biflavonoids as influenza H1N1 virus neuraminidase inhibitors. Eur. J. Med. Chem. 2010, 45, 1724-1730.

38. Li, Q.; Qi, J.; Zhang, W.; Vavricka, C.J.; Shi, Y.; Wei, J.; Feng, E.; Shen, J.; Chen, J.; Liu, D.; et al. The 2009 pandemic H1N1 neuraminidase N1 lacks the 150-cavity in its active site. Nat. Struct. Mol. Biol. 2010, 17, 1266-1268.

39. Grienke, U.; Schmidtke, M.; von Grafenstein, S.; Kirchmair, J.; Liedl, K.R.; Rollinger, J.M. Influenza neuraminidase: A druggable target for natural products. Nat. Prod. Rep. 2012, 29, 11-36.

40. Nervall, M.; Hanspers, P.; Carlsson, J.; Boukharta, L.; Åqvist, J. Predicting binding modes from free energy calculations. J. Med. Chem. 2008, 51, 2657-2667. 
41. Pedretti, A.; Villa, L.; Vistoli, G. VEGA-An open platform to develop chemo-bio-informatics applications, using plug-in architecture and script programming. J. Comput. Aided Mol. Des. 2004, 18, 167-173.

42. Li, Z.; Wan, H.; Shi, Y.; Ouyang, P. Personal experience with four kinds of chemical structure drawing software: Review on chemdraw, chemwindow, ISIS/draw, and chemsketch. J. Chem. Inf. Comput. Sci. 2004, 44, 1886-1890.

43. Xu, X.; Zhu, X.; Dwek, R.A.; Stevens, J.; Wilson, I.A. Structural characterization of the 1918 influenza virus H1N1 neuraminidase. J. Virol. 2008, 82, 10493-10501.

44. Trott, O.; Olson, A.J. Autodock vina: Improving the speed and accuracy of docking with a new scoring function, efficient optimization, and multithreading. J. Comput. Chem. 2010, 31, 455-461.

45. Durrant, J.D.; McCammon, J.A. NNScore 2.0: A neural-network receptor-ligand scoring function. J. Chem. Inf. Model. 2011, 51, 2897-2903.

46. Phillips, J.C.; Braun, R.; Wang, W.; Gumbart, J.; Tajkhorshid, E.; Villa, E.; Chipot, C.; Skeel, R.D.; Kalé, L.; Schulten, K. Scalable molecular dynamics with NAMD. J. Comput. Chem. 2005, 26, 1781-1802.

47. Bren, U.; Hodošček, M.; Koller, J. Development and validation of empirical force field parameters for netropsin. J. Chem. Inf. Model. 2005, 45, 1546-1552.

48. Schmidt, M.W.; Baldridge, K.K.; Boatz, J.A.; Elbert, S.T.; Gordon, M.S.; Jensen, J.H.; Koseki, S.; Matsunaga, N.; Nguyen, K.A.; Su, S.; et al. General atomic and molecular electronic structure system. J. Comput. Chem. 1993, 14, 1347-1363.

49. Dupradeau, F.-Y.; Pigache, A.; Zaffran, T.; Savineau, C.; Lelong, R.; Grivel, N.; Lelong, D.; Rosanski, W.; Cieplak, P. The R.E.D. tools: Advances in RESP and ESP charge derivation and force field library building. Phys. Chem. Chem. Phys. 2010, 12, 7821-7839.

50. Ryckaert, J.-P.; Ciccotti, G.; Berendsen, H.J.C. Numerical integration of the cartesian equations of motion of a system with constraints: Molecular dynamics of n-alkanes. J. Comput. Phys. 1977, 23, 327-341.

51. Case, D.A.; Cheatham, T.E.; Darden, T., III; Gohlke, H.; Luo, R.; Merz, K.M. Onufriev, J.A.; Simmerling, C.; Wang, B.; Woods, R. The Amber biomolecular simulation programs. J. Computat. Chem. 2005, 26, 1668-1688.

52. Wallace, A.C.; Laskowski, R.A.; Thornton, J.M. LIGPLOT: A program to generate schematic diagrams of protein-ligand interactions. Protein Eng. 1995, 8, 127-134.

53. Naïm, M.; Bhat, S.; Rankin, K.N.; Dennis, S.; Chowdhury, S.F.; Siddiqi, I.; Drabik, P.; Sulea, T.; Bayly, C.I.; Jakalian, A.; et al. Solvated interaction energy (SIE) for scoring protein-ligand binding affinities. 1. exploring the parameter space. J. Chem. Inf. Model. 2007, 47, 122-133.

(C) 2012 by the authors; licensee MDPI, Basel, Switzerland. This article is an open access article distributed under the terms and conditions of the Creative Commons Attribution license (http://creativecommons.org/licenses/by/3.0/). 